\title{
RELEASE KINETICS FROM NANO-INCLUSION-BASED AND AFFinity-BASEd Hydrogels: A CoMPARATIVE STUDY
}

\author{
P-L. Latreille ${ }^{a \dagger}$, S. Alsharif ${ }^{a b \dagger}$, O. Gourgas ${ }^{\text {c\#, }}$, S.F. Tehrani ${ }^{c}$, V.G. Roullin ${ }^{c}$ and X. \\ Banquy $^{a^{*}}$
}

${ }^{a}$ Canada Research Chair in Bio-inspired materials and Interfaces, Université de Montréal, PO Box 6128, succursale Centre-ville, Montreal QC, H3C 3J7, Canada.

${ }^{b}$ Faculty of pharmacy, Umm AlQura University, Al Taif Road, Mecca 24382, Saudi Arabia.

${ }^{c}$ Pharmaceutical Nanotechnology Laboratory, Faculty of Pharmacy, Université de Montréal, PO Box 6128, succursale Centre-ville, Montreal QC, H3C 3J7, Canada.

$\dagger$ These authors contributed equally to this work.

\# Present affiliation: Department of Mining and Materials Engineering, McGill University, 3610 University Street, Wong Building. Montreal, QC H3A 2B2

*to whom correspondence should be addressed: xavier.banquy@umontreal.ca

\begin{abstract}
In this study, we compare the release mechanisms from nanocomposite hydrogels. Liposomes made of different compositions of 1,2-dioleoyl-sn-glycero-3-phosphocholine (DOPC) and 1,2-dihexadecanoyl-snglycero-3-phosphocholine (DPPC), nanogels made of chitosan-hyaluronic acid association and crosslinked nanogels made of $\mathrm{N}$-isopropylacrylamide (NIPAM) and different ratios of methacrylic acid (MAA) were embedded in acrylamide hydrogels with a model drug, either sulforhodamine B or rhodamine 6G. Liposomes demonstrated the capacity to release their payload over 10 days while NIPAM nanogels and chitosan nanogels released within one or two days. We found that liposomes embedded in hydrogels presented two distinctive release mechanisms, a diffusive burst and a slower "sub-diffusive" release. Both nanogels on the other side presented no observable nor defined affinity-based release mechanism due to presence of salts, completely screening electrostatic interactions. The present work highlights critical points related to the release mechanisms from nanocomposite hydrogels as drug delivery devices or as biomedical tools for tissue engineering or regenerative medicine.
\end{abstract}




\section{Nano-inclusion-based release mechanism}

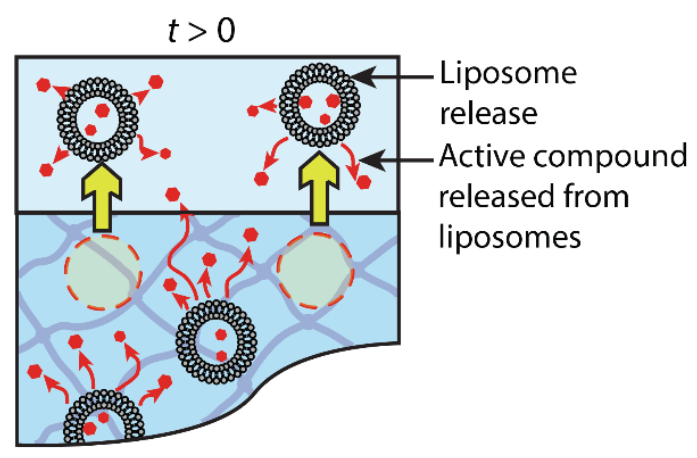

Slow release

\section{Affinity-based release mechanism}

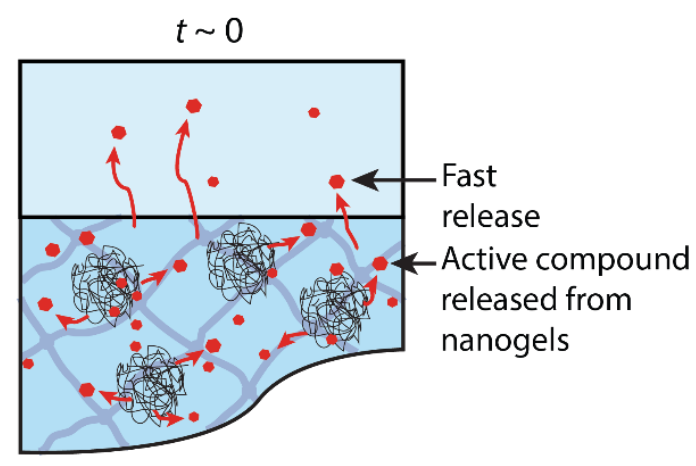

Fast release

Keywords: Nanocomposite hydrogel, release mechanism, liposome, nanogel, nano-structured hydrogel

\section{Highlights:}

- Nanostructured hydrogels with nano-inclusions or with affinity nano-domains are proposed as drug release systems

- Nano-inclusions and affinity domains are finely tuned and well characterized

- Key release mechanisms were identified for nano-inclusion-based hydrogels

- Affinity-based hydrogels are strongly affected by physiologically relevant media 


\section{Introduction}

Pharmaceutical science is recently taking a path where engineered systems for drug delivery are increasing in complexity. However, over the past two decades, significant advancements have occurred in the field of nanotechnology that have led to the development of nanoscaled particles with specific physical and chemical properties beneficial for therapeutic purposes [1]. Nanoparticles were first engineered as drug controlled-release systems to facilitate administration and improve therapeutic efficiency. Yet, more recently, nanoparticles have started being used along with hydrogels as novel biocompatible drug delivery platforms as well as innovative biomaterials for tissue engineering and regenerative medicine, generally referred to as nanocomposite hydrogels. Indeed, nanoparticles can provide adhesion or affinity domains [2], nano-inclusions [3], confer stimulus-responsiveness [4] or increase mechanical properties of the gel $[5]$.

Basically, these new features mostly depend on nanoparticles-hydrogel combinations and can lead to greater flexibility in design such as interpenetrated networks, nano-domains within the hydrogel or in organized scaffolds. The complexity and stimulus-responsiveness of nanocomposite hydrogels might open new versatile and tunable controlled drug delivery devices. These nano-domains can add specific ability to use triggers or stimuli to achieve a desired response which in some cases can react faster than in the bulk [6-8]. Therefore, they are highly relevant for a broad range of applications from pharmaceutical (patches, implants or tissue engineered platforms) [9, 10] to environmental applications (chemical removal) [11]. Additionally, their modified mechanical properties enhanced from nanoparticles would also make them an appealing material for skin or cartilage replacement [5].

While the complexity degree of these materials is increasing, it gets harder to define which composition is better or optimal for a specific purpose. In this study, we focused toward the comparison of nanocomposite hydrogels of various natures to achieve a controlled delivery of an active compound. For this purpose, we used three types of nanoparticles, i.e. liposomes, chitosan nanogels and NIPAM nanogels, embedded in hydrogels to create nano-inclusions or affinity domains. Indeed, the use of nano-inclusions from liposomes 
to nanogels have been suggested via broad range of applications [12]. However, nanogel nano-inclusions have been mostly reported for their capacity to tune hydrogel mechanical properties [8]. Only a few studies report their ability to control the release of a molecule [13-16].

Liposomes are often regarded as one of the most successful nanocarriers because of their ability to provide controlled release, prolonged effect and temperature sensitivity [17-19]. We used liposomes as nanoinclusions and as a typical diffusion-based release system because of their structure made from a phospholipid bilayer and different phospholipid compositions, as first described by Alec Bangham [17].

Crosslinked gel nanoparticles based on poly N-isopropylacrylamide (pNIPAM) and N,N'-methylenebis(acrylamide) (bisA) copolymers also have drawn interest for their thermosensitive and $\mathrm{pH}$-sensitive features [20]. Introduced by Pelton and Chibante by polymerization of NIPAM and BisA [21], these crosslinked gel nanoparticles can also include a negative charge in their polymeric structure with the copolymerization of NIPAM and methacrylic acid (MAA). However, MAA inclusion alters some of the nanoparticle properties such as lowering the critical solubility temperature (LCST) and pH-responsiveness [22-24]. In this report, embedded NIPAM nanogels (200-600 nm) in hydrogels were used to create affinity and responsive domains.

Finally, we also studied nanogels made from the ionic gelation of polycationic chitosan with polyanionic hyaluronic acid (HA) and sodium tripolyphosphate (TPP) due to their bioactivity, biodegradability and biocompatibility that make them ideal material for in vivo applications [25]. Their components are biorelevant and possess a highly-charged matrix from natural polyelectrolytes [26-30] which was found to be advantageous in terms of active compound delivery [31] and regenerative medicine [32].

Controlling the release of an active compound among drug delivery devices is mandatory since invasive administration routes in almost every circumstance would benefit from sustained release by reducing dosage frequency and improving current treatments. For instance, implants, drugloaded patches and tissue-designed platforms are potential applications of nanocomposite hydrogels 
but require a biocompatible polymeric matrix to entrap particles capable of sustaining and controlling the release of the active substance $[33,34]$. In order to produce these nanocomposite hydrogels, a model hydrogel of acrylamide/bisA with high water content was used to represent biocompatible or tissue-mimicking synthetic matrices for the embedment of nanocarriers. The combined structure of different nanogels and the acrylamide hydrogel result in a semiinterpenetrated network as already reported [7]. The objectives of this study were to compare all three particle formulations in their capacity to control the release of a model compound. Previous studies have focused on describing the release mechanism of plain hydrogels as a diffusion-based or affinity-based release system and recent reports show interesting mathematical model to describe the release mechanism $[35,36]$. However, such works, to our knowledge, have never been translated to embedded particles within hydrogel networks with the objective to compare both affinity-based and diffusion-based nano-inclusions. Also, we highlight the release of liposomal particles which have never been considered prior to this study. Since the nano-inclusions used in this study have different stimulus-responsiveness, the impact of temperature, medium salinity and composition were also assessed. Thus, the comparison of these three different nanocarriers embedded in hydrogels contributes to the understanding of the various release mechanisms found with those complex systems and illustrates how their structure can influence their release behavior.

\section{Materials and methods}

\subsection{Chemicals and reagents}

All chemicals and reagents were of analytical grade and used without further purifications. Phospholipids including 1,2-Dioleoyl-sn-glycero-3-phosphocholine (DOPC) and 1,2dihexadecanoyl-sn-glycero-3-phosphocholine (DPPC) were purchased from Avanti Polar Lipids (Alabaster, AL, USA). Surfactant triton X-100, Sephadex ${ }^{\circledR}$ G-50, acrylamide, NIsopropylacrylamide (NIPAM), N,N'-methylene-bis(acrylamide) (BisA), methacrylic acid (MAA) with 250 ppm inhibitor MEHQ, sodium dodecyl sulfate (SDS) approx 95\% and 4-(2-hydroxyethyl)- 
1-piperazineethanesulfonic acid (HEPES) were purchased from Sigma-Aldrich (Oakville, ON, Canada). Chitosan (Mw $=88 \mathrm{kDa})$ derived from shrimp shells and hyaluronic acid sodium salt $(\mathrm{Mw}$ $=1400 \mathrm{kDa}$ ) extracted from Streptococcus equi sp were purchased from Sigma (Oakville, ON, Canada). Sodium tripolyphosphate (TPP) and Kiton Red S (Sulforhodamine B) were purchased from Alfa Aesar (Ward Hill, MA, USA). Rhodamine 6G was acquired from Acros Organics (New Jersey, USA). Citric acid was purchased from Anachemia (Canada). Ammonium persulfate (APS) was from Fisher-Biotech (Canada). Irgacure ® 2959 was supplied by BASF (Switzerland).

\subsection{Preparation of formulations}

\subsubsection{Liposome preparation}

Preparation of liposomes was adapted from Monnard et al [37]. $20 \mathrm{mg}$ of (DOPC:DPPC) mixture were dissolved in $1 \mathrm{~mL}$ of chloroform in order to prepare three different batches with DOPC:DPPC molar ratios of (50:50), (60:40), and (70:30). Then, the solvent was slowly removed by rotary evaporation to form a thin lipid film at the bottom of the flask. The lipid film was dried under vacuum to eliminate the residual solvent content. $1 \mathrm{~mL}$ of sulforhodamine B (30 mM) in HEPES buffer ( $5 \mathrm{mM}$ HEPES with $150 \mathrm{mM} \mathrm{NaCl}$ ) was added to the flask to hydrate the lipid film at $60^{\circ} \mathrm{C}$ while mixing and vortexing until complete hydration. The dispersion was then treated by 10 freezethaw cycles followed by 21 cycles of extrusion (Avanti Mini-Extruder; Avanti Polar Lipids, USA) through a $200-\mathrm{nm}$ then a $100-\mathrm{nm}$ pore size polycarbonate membrane. Non-encapsulated sulforhodamine B was removed from the liposomes by size exclusion chromatography with Sephadex ${ }^{\circledR}$ G-50 stationary phase and a HEPES buffer mobile phase.

\subsubsection{Chitosan nanogel preparation}

The chitosan nanogel formulation was prepared as described by Courant et al [38]. Briefly, CS was solubilized in a $10 \%(\mathrm{w} / \mathrm{v})$ citric acid aqueous solution $(9 \mathrm{~mL}, \mathrm{pH}=2.2)$ at a concentration of 2.5 $\mathrm{mg} / \mathrm{mL}$ under magnetic stirring until complete dissolution. A solution of TPP $(1.2 \mathrm{mg} / \mathrm{mL})$ and HA 
$(0.8 \mathrm{mg} / \mathrm{mL})$ was prepared in $4.5 \mathrm{~mL}$ Milli-Q water. Both solutions were filtered through $0.2 \mu \mathrm{m}$ nylon membrane filter (Ultident Scientific, Canada). Sulforhodamine B (4 mg/mL) was then added to the TPP/HA solution. Subsequently, the TPP/HA solution was added dropwise to the CS solution on an ice bath and under ultrasonication (Fischer Scientific Sonic Dismembrator F550 Ultrasonic Homogenizer; power sonicator 20\%) for 90 seconds. This process rapidly formed inter- and intramolecular electrostatic-mediated crosslinks between the polyanions and the protonated CS chains inducing nanoparticle formation [39]. The resulting turbid suspension was kept under magnetic stirring for 15 additional minutes to allow strengthening of the formed nanogels. The resulting loaded nanogels were stored at $4{ }^{\circ} \mathrm{C}$ and protected from light exposure. Loaded nanogel suspensions $(12 \mathrm{~mL})$ were purified three times at room temperature against 1.2 L of HEPES buffer by the tangential flow filtration method using MicroKros ${ }^{\circledR}$ hollow fiber modules (Spectrum, MicroKros® ME, MWCO $0.05 \mu \mathrm{m})$.

\subsubsection{NIPAM-co-MAA nanogel preparation}

Thermosensitive NIPAM nanogels were prepared to provide an anionic charge (MAA) within its structure (NIPAM-BisA) with different charge ratios. The synthesis of the NIPAM-co-MAA particles was carried on with a single-step synthesis, following multiple published formulation and synthesis protocols [23, 40-42]. NIPAM and MAA were dissolved in degassed MilliQ water at different molar ratios $\left(\mathrm{mol}_{\mathrm{MAA}} /\left[\mathrm{mol}_{\mathrm{NIPAM}}+\mathrm{mol}_{\mathrm{MAA}}\right]\right)$, see table 1 . Then, BisA at $5.3 \%$ molar ratio $\left(\mathrm{mol}_{\mathrm{MAA}} /\left[\mathrm{mol}_{\mathrm{NIPAM}}+\mathrm{mol}_{\mathrm{MAA}}+\mathrm{mol}_{\mathrm{BisA}}\right]\right)$ and SDS at $867 \mu \mathrm{mol} / \mathrm{L}$ were dissolved in the degassed solution. A total of $150 \mathrm{~mL}$ of monomer/crosslinker/surfactant solution was transferred into a threenecked flask heated under reflux at approximately $60^{\circ} \mathrm{C}$ with constant Argon gas flow and mechanical stirring $(275 \mathrm{rpm})$. The reaction was initiated with $10 \mathrm{~mL}$ APS (solution at $2.9 \mathrm{mmol} / \mathrm{L}$ ) degassed by vacuum, while slowly increasing temperature at $75^{\circ} \mathrm{C}$ and maintaining argon flow rate and mechanically stirring the reacting solution. The reaction was stopped after $4.5 \mathrm{~h}$ by cooling down the particle suspensions at room temperature. The synthesized NIPAM nanogels were 
purified in batches of $70 \mathrm{~mL}$ by two consecutive dynamic dialysis against $20 \mathrm{~L}$ MilliQ water for 16 hours and 4 hours respectively, using Spectra/Por ${ }^{\circledR}$ Tube-A-Lyzer ${ }^{\circledR}$ (Rancho Dominguez, USA) with $100 \mathrm{kDa}$ MWCO cellulose ester membrane. The nanogel suspensions were stored at $4^{\circ} \mathrm{C}$ until further use.

\subsection{Nanoparticle Imaging}

\subsubsection{Atomic force microscopy imaging}

NIPAM nanogels (NIPAM-co-MAA10\%) and chitosan nanogels $(10 \mu \mathrm{L})$ were deposited on mica surfaces at a concentration of $25 \mu \mathrm{g} / \mathrm{mL}$ and $2.5 \mu \mathrm{g} / \mathrm{mL}$ respectively. The samples were left to dry then imaging was carried out using a Multimode Dimension 3100 AFM equipped with a NanoScope VIII controller (Digital Instruments) in the peak force QNM mode. A Scanasyst-air tip (silicon tip on nitride lever) was used for imaging the nanoparticles. The nanoparticles were imaged first on a large square area of $(10 \times 10 \mu \mathrm{m}$ or $3 \times 3 \mu \mathrm{m})$, then a single nanoparticle was imaged $(1 \times 1 \mu \mathrm{m})$ at 256 scans per line resolution.

\subsubsection{Fluorescence microscopy imaging}

A volume of $10 \mu \mathrm{L}$ of NIPAM nanogels and liposomes were deposited on a clean glass slide and covered with a cover slit. The sample was imaged using an inverted microscope (Olympus IX81) equipped with a Retiga 2000R CCD camera from QImaging (Surrey, Canada). The images were acquired at a 100× magnification using typical Texas Red filter set. Images were cropped to specifically select an area containing sufficient quantity of nanoparticles. Color balance was adjusted with ImageJ software (version 1.50b).

\subsection{Physicochemical characterization of formulations}

The size of liposomes, chitosan nanogels and NIPAM nanogels were measured using dynamic light scattering (DLS) using a Malvern Zetasizer NanoZS (Malvern Instruments, UK) and expressed as 
Z-averages of the hydrodynamic diameters and polydispersity indexes (PdI). Each sample was analyzed in triplicate at $25^{\circ} \mathrm{C}$ unless otherwise specified. Water and water-citric acid $(0.48 \mathrm{M})$ mixtures were used as reference dispersing media for liposomes and nanogels respectively. The NIPAM nanogel size was measured at different temperatures to characterize their thermosensitivity. Measurement started from $20^{\circ} \mathrm{C}$, increasing with $2^{\circ} \mathrm{C}$ increments between each measurement, up to $40^{\circ} \mathrm{C}$ in MilliQ water, phosphate buffered saline at $\mathrm{pH}$ 7.4 (PBS) and HEPES 5mM without salt (pH 7.4). Zeta potential was acquired by measurement of electrophoretic mobility at $25^{\circ} \mathrm{C}$, in triplicate for each sample (Malvern Zetasier Nano-ZS, $150 \mathrm{~V}$ ) in MilliQ water for liposomes and nanogels. Zeta potentials of NIPAM nanogels were estimated in $4 \mathrm{mM} \mathrm{NaCl}$ medium at $22^{\circ} \mathrm{C}$ and $38^{\circ} \mathrm{C}$ (Malvern Zetasier Nano-ZS, 150 V).

\subsection{Determination of loading efficiency (LE\%) and drug loading (DL\%)}

The loading efficiencies (LE\%) and the drug loadings (DL\%) of all formulations were calculated as follows in equations 1 and 2 respectively:

$L E \%=\frac{D_{\text {in }}}{D_{\text {total }}} \times 100$

$D L \%=\frac{D_{\text {in }}}{D_{\text {in }}+N P} \times 100$

Where $D_{\text {in }}$ is the amount (mass) of the dye loaded in the nanoparticles, $D_{\text {total }}$ is the total amount of the dye introduced in the system, and NP is the amount of nanoparticles.

\subsubsection{Liposomes}

The Bartlett assay was performed to quantify the total inorganic phosphates of phospholipids, thus the total phospholipid content. A known volume collected from each batch of liposomes loaded with sulforhodamine B was appropriately diluted in HEPES buffer. The amount of loaded sulforhodamine B in liposomes was determined by spectrofluorimetry in triplicate (Hitachi F-2710 fluorescence spectrophotometer) through addition of $10 \mu \mathrm{L}$ of surfactant Triton $\mathrm{X}-100$. The 
sulforhodamine B fluorescence intensity was measured at $582 \mathrm{~nm}$ from an excitation wavelength of $563 \mathrm{~nm}$ at room temperature. DL\% and LE\% were thereafter calculated as described in equation 1 and 2.

\subsubsection{Chitosan nanogels}

Sulforhodamine B-loaded nanogels were centrifuged at $20,000 \mathrm{rpm}, 4^{\circ} \mathrm{C}$ for 90 minutes to remove nanogels from the aqueous suspension medium. The supernatant was collected and the amount of free sulforhodamine $\mathrm{B}$ in the supernatant was determined by fluorescence quantification, as abovementioned. Therefore, the amount of encapsulated sulforhodamine B was determined by indirect dosage and the mass of nanogels collected by freeze-drying (triplicate). Fluorescence intensity measurements were performed using the same parameters as with liposomes.

\subsubsection{NIPAM-co-MAA nanogels}

NIPAM nanogel concentration in stock solution was determined by freeze-drying after purification and weighting the residual polymer. Suspensions were loaded using R6G, a positively charged fluorescent dye which was expected to interact with the negatively-charged NIPAM-co-MAA nanogels. Stock solutions of NIPAM nanogels and R6G were prepared separately in milliQ water at $0.5 \mathrm{mg} / \mathrm{mL}$ and kept at $4^{\circ} \mathrm{C}$ until use. Loading occurred by incubating $\mathrm{R} 6 \mathrm{G}(20,40,60,80 \mu \mathrm{g} / \mathrm{mL})$ in presence of $100 \mu \mathrm{g} / \mathrm{mL}$ of NIPAM nanogels in MilliQ water or in another incubation medium, such as HEPES without salt (pH 7.4) or HEPES with $145 \mathrm{mM} \mathrm{NaCl}(\mathrm{pH} 7.4)$. The suspensions were vortexed and then incubated for 1 hour at two different temperatures $\left(25^{\circ} \mathrm{C}\right.$ and $\left.40^{\circ} \mathrm{C}\right)$ or temperature cycles. Typically, the cycle started at the swollen-state temperature $\left(0^{\circ} \mathrm{C}\right.$ or $\left.25^{\circ} \mathrm{C}\right)$ for 20 minutes then the suspensions were heated to $40^{\circ} \mathrm{C}$ for 20 minutes and finally returned to their initial temperature for the last 20 minutes. At the end of the incubation process, the drug-loaded NIPAM nanogels were centrifuged at 25,000 g for 30 minutes, at room temperature. Supernatants were collected then diluted 20 times. Free R6G was quantified by fluorescence, thus indirectly 
quantifying the loaded amount. Each parameter in this study was assessed in independent triplicate $(\mathrm{n}=3)$. DL\% and LE\% were calculated with equation 1 and 2, respectively.

\subsection{Hydrogel preparation}

Hydrogels were prepared by free radical photopolymerization of acrylamide and N-N'-methylenebisacrylamide (BisAc) in HEPES buffer (unless otherwise specified) with a cross-linker / monomer molar ratio of 5\% [43]. The cross-linker/monomer solution was prepared at $95 \mathrm{mg} / \mathrm{mL}$ of acrylamide and $5 \mathrm{mg} / \mathrm{mL} \mathrm{N}-\mathrm{N}$ '-methylene-bisacrylamide in HEPES buffer. The stock solution was frozen at $-80^{\circ} \mathrm{C}$, degassed under vacuum for 30 minutes, then thawed and $2 \mathrm{~mL}$ of this solution was transferred into a 20-mL glass beaker. Irgacure ${ }^{\circledR} 2959(2.9 \mathrm{mg} / \mathrm{mL}$ ) in HEPES buffer (unless otherwise specified), also degassed, was added as a $300-\mu \mathrm{L}$ polymerization mixture in the beaker. For liposome-, chitosan nanogel- and NIPAM nanogel- embedded hydrogels, the quantity of sulforhodamine B or R6G (loaded in particles) was adjusted at $270 \mu \mathrm{g}$ per hydrogel batch. The final solution, $2.4 \mathrm{~mL}$ in total, was completed with either nanoparticle solution containing the dye or with water. The hydrogels were photopolymerized, covered with a glass slide, under UV lamp for 5-40 minutes of exposure (365 nm; UVP Mercury Spot Low, 100MW Longwave).

\subsection{Release study from hydrogels}

Photopolymerized hydrogel disks were weighted and 5-mm diameter cylinders were sampled using a biopsy punch (Miltex $\left.{ }^{\circledR}\right)$. Thereafter, those pieces were accurately weighted to calculate the sulforhodamine B content in every piece. Those hydrogel samples were individually positioned in $10 \mathrm{~mL}$ of HEPES buffer (pH 7.4) at $4^{\circ} \mathrm{C}$ and $37^{\circ} \mathrm{C}$. At predetermined times, the incubation medium was entirely sampled and replaced by fresh HEPES buffer. The sampled medium was kept at $4{ }^{\circ} \mathrm{C}$ in darkness until further analysis. $200-\mu \mathrm{L}$ aliquots were analyzed by spectrofluorimetry (Tecan Safire Monochromatic Fluorescence) for each time-point. Each condition was studied in triplicate 
$(n=3)$. The released dye percentage was calculated as the ratio of the cumulative released quantity and the quantity in the 5-mm hydrogel samples.

\section{Results and Discussion}

\subsection{Preparation and characterization of liposomes}

Table 1. Composition, physicochemical properties and loading characterization of formulations

\begin{tabular}{|c|c|c|c|c|c|c|c|}
\hline $\begin{array}{c}\text { Formulation } \\
\text { design }\end{array}$ & Composition & $\begin{array}{c}\text { Particle Size, } \\
\text { PS ** } \\
(\mathrm{nm})\end{array}$ & $\begin{array}{c}\text { Polydispersity } \\
\text { Index, PDI } \\
\text { (a.u) }\end{array}$ & $\begin{array}{c}\text { Zeta Potential, } \\
\text { ZP } \\
(\mathrm{mV})\end{array}$ & $\begin{array}{c}\text { Loading } \\
\text { Efficiency, LE } \\
(\%)\end{array}$ & $\begin{array}{c}\text { Drug Loading, } \\
\text { DL } \\
(\%) \\
\end{array}$ & Drug model \\
\hline \multirow[t]{3}{*}{ Liposome } & $\begin{array}{c}\text { DOPC:DPPC } \\
50: 50\end{array}$ & $132 \pm 2$ & 0.10 & $-24.0 \pm 4.0$ & 8 & 40 & \multirow{4}{*}{$\begin{array}{c}\text { Sulforhodamine } \\
\text { B } \\
\text { (SRB) }\end{array}$} \\
\hline & $\begin{array}{c}\text { DOPC:DPPC } \\
60: 40\end{array}$ & $140 \pm 1$ & 0.10 & $-57.7 \pm 1.7$ & 5 & 27 & \\
\hline & $\begin{array}{c}\text { DOPC:DPPC } \\
70: 30\end{array}$ & $133 \pm 1$ & 0.07 & $-60.7 \pm 1.6$ & 1 & 12 & \\
\hline Nanogel & HA + Chitosan & $195 \pm 0$ & 0.2 & $+46.3 \pm 2.6$ & 96 & 35 & \\
\hline \multirow{6}{*}{$\begin{array}{c}\text { NIPAM } \\
\text { nanogel* }\end{array}$} & NIPAM & $203 \pm 2$ & 0.05 & $-1.8 \pm 0.1$ & 0.7 - N.D. & 0.4 - N.D. & \multirow{6}{*}{$\begin{array}{c}\text { Rhodamine 6G } \\
\text { (R6G) }\end{array}$} \\
\hline & $\begin{array}{c}\text { NIPAM-co-MAA } \\
5 \%\end{array}$ & $290 \pm 4$ & 0.08 & $-6.3 \pm 0.1$ & $1.3-3.5$ & $0.8-2.1$ & \\
\hline & $\begin{array}{c}\text { NIPAM-co-MAA } \\
10 \%\end{array}$ & $356 \pm 4$ & 0.04 & $-7.0 \pm 0.2$ & $10.4-38.3$ & $5.9-18.7$ & \\
\hline & $\begin{array}{c}\text { NIPAM-co-MAA } \\
12.5 \%\end{array}$ & $496 \pm 2$ & 0.12 & $-7.2 \pm 0.3$ & $8.5-44.7$ & $4.9-21.2$ & \\
\hline & $\begin{array}{c}\text { NIPAM-co-MAA } \\
15 \%\end{array}$ & $501 \pm 10$ & 0.12 & $-8.9 \pm 0.1$ & $13.9-49.3$ & $7.7-22.8$ & \\
\hline & $\begin{array}{c}\text { NIPAM-co-MAA } \\
20 \%\end{array}$ & $512 \pm 15$ & 0.08 & $-10.5 \pm 0.4$ & $14.6-50.6$ & $8.1-23.3$ & \\
\hline
\end{tabular}

* Loading efficiencies and drug loading for NIPAM nanogels are given by loading in milliQ water and HEPES 5mM respectively (water - HEPES). ** Means of three Z-average values and their standard deviation.

Three formulations with different phospholipid molar ratios (50:50, 60:40, and 70:30) were prepared to identify the effect of liposome composition on particle size and zeta potential (Table 1). Increasing DOPC concentration in the phospholipid mixture had a minor effect on the hydrodynamic diameters among all formulations with stable PdI values $<0.1$ (Table 1). However, $\mathrm{ZP}$ values were found to increase significantly from $-24.0 \pm 4.0 \mathrm{mV}$ in $50: 50$ formulations to -60.7 $\pm 1.6 \mathrm{mV}$ along with the increase in DOPC. 
As depicted in figure 1C (left panel), liposomes appeared as small dots. Larger fluorescence spots correspond to aggregated liposomes on the surface of the glass slide. Table 1 shows that highest LE\% (40\%) and EE\% (8\%) were obtained in the formulation that exhibit equal ratios of DOPC:DPPC (50:50), while the lowest LE\% (12\%) and EE\% (1\%) were obtained from the formulation of (70:30) where DOPC content was the highest. Therefore, these findings suggest that (50:50) formulation is the optimal formulation to achieve the best sulforhodamine B-loaded liposomes. These variations in $\mathrm{LE} \%, \mathrm{EE} \%$, and $\mathrm{ZP}$ are due to the changes in the liposome membrane fluidity that depends on differences in both phospholipid composition and transition temperature among the three formulations. Since DOPC and DPPC have transition temperatures of $-21^{\circ} \mathrm{C}$ and $41^{\circ} \mathrm{C}$ (as provided by the manufacturer), respectively, DOPC is responsible for the fluidity and DPPC the rigidity. Owing to the presence of a double bond in the acyl chain of DOPC, increasing the DOPC:DPPC ratio in the formulations provided liposomes with higher bilayer membrane fluidity. Therefore, the liposomes with higher DOPC composition (60:40, 70:30) were not capable to encapsulate sufficient amounts of sulforhodamine B compared to liposomes with higher DPPC (50:50), due to the constant leakage of the model drug during the nanosystem formation. The same explanation applies to decreasing ZP values with higher DOPC composition.

\subsection{Chitosan nanogel characterization}

The prepared formulation of chitosan and HA nanogels loaded with surforhodamine B was initially characterized in terms of particle size and zeta potential (Table 1). The nanogels were small (mean hydrodynamic diameter $\approx 200 \mathrm{~nm}$ ) and the particle size showed a larger distribution than for liposomes $(\mathrm{PdI}=0.2)$. The zeta potential indicates a strong cationic charge $(\mathrm{ZP}=46.3 \pm 2.6 \mathrm{mV})$ on the surface of the nanogels. These findings corroborate with other reported nanogels using similar compositions $[44,45]$ thus providing interesting insights about its internal structure. Indeed, from these results, they seem structured in a way that HA chains mainly constitute the nanogel core, 
whereas chitosan chains predominate at the nanogel surface, exposing their positive charges. This assumption is supported by other reports where it was observed that, to produce a negative surface charge for chitosan/HA nanogels, one has either to increase drastically the HA content [46] or to coat chitosan-based preformed nanogels with HA chains [47].

The DL and LE of sulforhodamine B were also evaluated in nanogels by indirect quantification of the supernatant resulting from the high-speed centrifugation of the nanogel suspensions. High LE was achieved, encapsulating almost all the fluorescent dye $(\mathrm{LE}=96 \%)$ and consequently achieving also high drug loading $(\mathrm{DL}=35 \%)$. The outer shell, highly concentrated in chitosan chains, as suggested by the measured ZP values, might have facilitated the retention of surlforhodamine B which carries a net negative charge in the loading conditions. This uneven distribution of polymer within the nanogel is also supported with observation of non spherical shape in AFM imaging (figure 1B, right panel).

\subsection{NIPAM-co-MAA nanogels characterization}

We have synthesized multiple chemically crosslinked nanogels using NIPAM as a reference structure in which we have incorporated different ratios of MAA ranging from 5\% to $20 \%$. Particle size (PS) and ZP were measured after purification of the suspensions. At $26^{\circ} \mathrm{C}$ as described in table 1, PS increased with increasing MAA content from $203 \mathrm{~nm}$ (MAA 0\%) to $512 \mathrm{~nm}$ (MAA 20\%), while PdI remained below 0.15 for each formulation, indicating narrow distribution in pure water. Similarly, ZP decreased slightly with increasing MAA ratios from $-6.3 \pm 0.1 \mathrm{mV}$ (MAA 5\%) up to $-10.5 \pm 0.4 \mathrm{mV}(\mathrm{MAA} 20 \%)$, while pure NIPAM nanogels remained mostly neutral $(\mathrm{ZP}=-1.8 \pm$ $0.1 \mathrm{mV}$ ) at $22^{\circ} \mathrm{C}$ (see table 1$)$. 
Thermosensitivity of NIPAM nanogels was also investigated between $20^{\circ} \mathrm{C}$ to $40^{\circ} \mathrm{C}$ for each $2^{\circ} \mathrm{C}$ increments. Increasing the ratio of MAA within the particles slightly shifted the LCST from $32^{\circ} \mathrm{C}$ to $36^{\circ} \mathrm{C}$

A
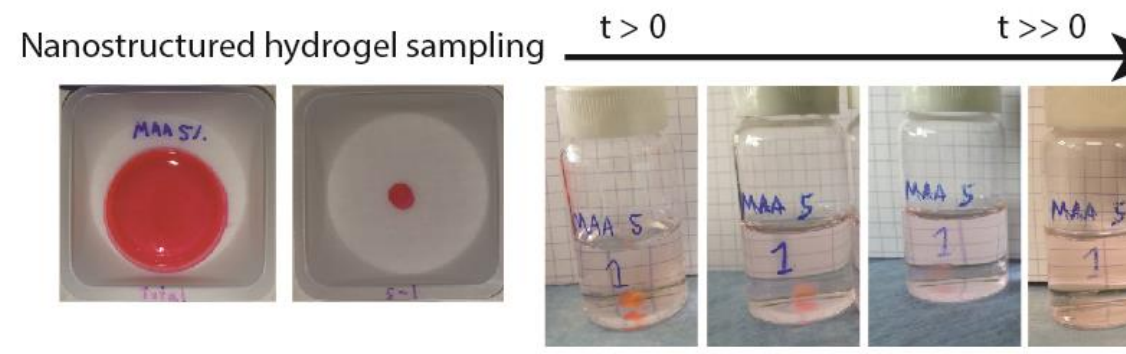

B

NIPAM-MAA $10 \%$

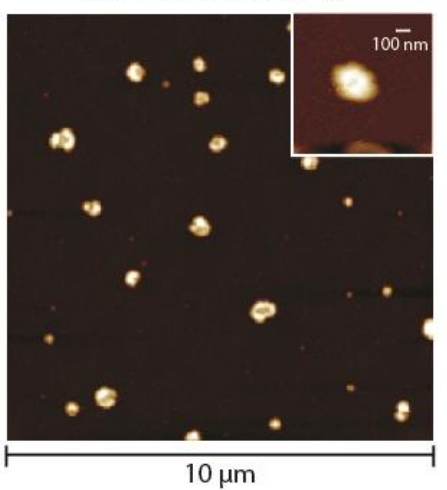

HA-Chitosan Nanogels

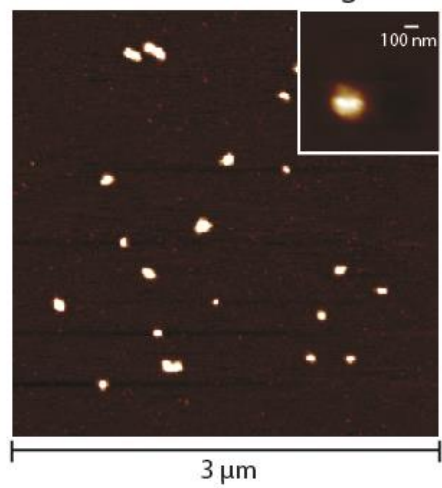

C

Liposomes loaded with sulforhodamine B

NIPAM-MAA 20\% loaded with rhodamine $6 \mathrm{G}$
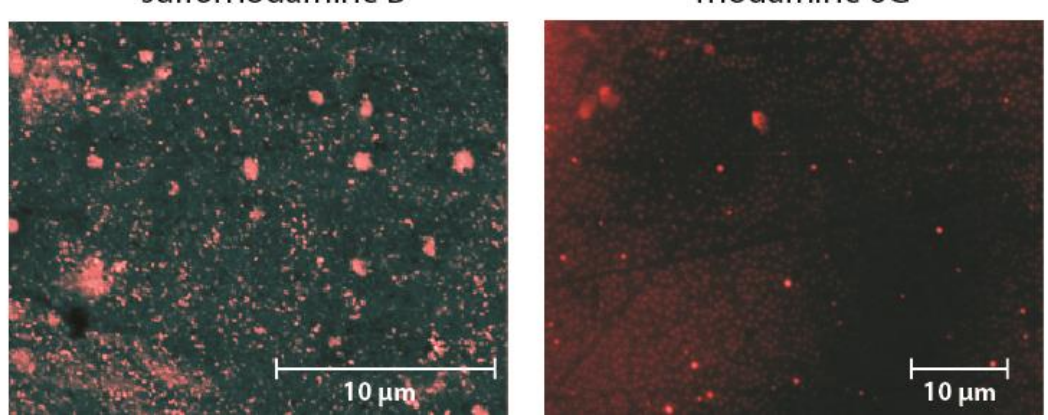

Figure 1. (A) Macroscopic visualization of the hydrogel structure (before and after sampling) and its evolution over time within the release medium. (B) AFM micrographs of NIPAM-coMAA 10\% and HA-chitosan nanogels. (C) Fluorescence micrograph of liposomes (loaded with SRB) in comparison with NIPAM-co-MAA 20\% nanogels (loaded with R6G) at 100X magnification. 
approximately, which was in accordance with other reports [48]. Collapsed state ( $T>$ LCST) was characterized by an approximated 50\% PS reduction compared to the swollen state $(T<$ LCST). Moreover, for MAA ratios higher than $12.5 \%$, a second collapse transition was observed between temperatures $22^{\circ} \mathrm{C}$ to $26^{\circ} \mathrm{C}$ (Fig S1). Since PdI values remains very low $(<0.12)$ for high MAA content in the nanogels, it suggests that MAA distribution in the particles is not homogeneous, thus creating richer domains that exhibit different thermodynamic properties and different LSCT. As observed with AFM (figure 1), NIPAM nanogels exhibit a near spherical shape (also supported by fluorescence imaging) with some irregularities which are potentially the result of an uneven MAA incorporation within the particle. At pH 7.4 medium (salt free), swollen/collapsed transition was very weak (12 to 16\% PS reduction for those observable). When salts were added $(145 \mathrm{mM} \mathrm{NaCl})$ to $\mathrm{pH}$ 7.4 PBS $10 \mathrm{mM}$, size transition from swollen to collapsed state was only slightly increased (18\% to $21 \%$ PS reduction). This particle size change is consistent with the collapse of pNIPAM above its LCST. Ionization of carboxylic acid groups (at pH 7.4) located inside the particle might impede the interaction of the isopropyl groups present in NIPAM to drastically reduce the dehydration of the particle core in the collapsed state. Typical pKa values of pMAA have been reported between 5 and 6 which also supports this hypothesis [49]. Therefore, at $\mathrm{pH} 7.4$, electrostatic repulsions within the particle are so effective that they nearly prevent the nanogel to collapse. The addition of salt to a $\mathrm{pH}=7.4$ weakly decrease the electrostatic repulsions of MAA which marginally increase the size ratio between swollen and collapsed states. Furthermore, the NIPAM without anionic functionalization aggregated in buffered medium $\mathrm{pH} 7.4$ without salt indicating that the ionic force from HEPES $5 \mathrm{mM}$ was sufficient to initiate the aggregation, which is an insight of a very weak colloidal stability [50].

On the other hand, the surface charge of the NIPAM nanogels was significantly influenced by temperature. It was observed that increasing the temperature from 22 to $38^{\circ} \mathrm{C}$ increased the $\mathrm{ZP}$ for all NIPAM nanogels from MAA 0\% (-1.8 to $-11.8 \mathrm{mV})$ to MAA $20 \%(-10.5$ to -16.3$)$ as shown in figure S2. This behavior can be explained considering that, upon collapse, MAA functional groups 
as well as the sulfated chain ends bearing the initiator are expelled from the core therefore increasing the surface charge [51].

\subsection{DL, LE and affinity characterizations of NIPAM-co-MAA nanogels}

To gain more insights into the parameters driving the loading mechanism carried through electrostatic interactions with MAA, the characterization of DL and LE as a function of MAA content in nanogels, $\mathrm{R} 6 \mathrm{G}$ / particle ratio, salt, $\mathrm{pH}$ and incubation temperature was performed (figure 2 and figure $\mathrm{S} 3$ ).

At first, NIPAM nanogels were incubated with different R6G concentrations for 1 hour at $25^{\circ} \mathrm{C}$ in pure water. DL was determined for each ratio of MAA within the nanogel as represented in figure 2A. Only nanogels without MAA did not demonstrate significant encapsulation of R6G regardless the R6G/particle ratio. Increasing MAA concentrations within the NIPAM nanogels proved to increase the R6G loading as well, up to $16.3 \pm 2.7 \%$ (DL) and $32.4 \pm 5.3 \%$ (LE) with $20 \%$ MAA at a R6G/nanogel ratio of 0.6 (figure 2A). Noticeably, a plateau was found from a ratio of 0.6 which can be attributed as the saturation of all affinity domains in the nanogels.

The effect of temperature and incubation medium on DL was also evaluated using one representative formulation (MAA 10\%). In HEPES $5 \mathrm{mM}(\mathrm{pH}=7.4)$ we observed an important amount of loaded $\mathrm{R} 6 \mathrm{G}(\mathrm{DL}=38.0 \pm 0.2 \%)$ in comparison with pure water $(\mathrm{DL}=6.9 \pm 1.6 \%$ at $\mathrm{pH}$ 5.5). This increase seems directly related to the ionization of MAA carboxylic groups discussed earlier. No peak or plateau was reached in terms of DL in HEPES $5 \mathrm{mM}$, suggesting that it is still possible to increase DL with a ratio R6G/particle higher than 0.8 (fig. S3). On the contrary, the presence of salts in PBS $10 \mathrm{mM}(\mathrm{NaCl} 145 \mathrm{mM}$ at $\mathrm{pH}=7.4)$ totally prevented the loading of $\mathrm{R} 6 \mathrm{G}$ into the NIPAM nanogel (Fig. 1B, light blue histograms), thus evidencing the electrostatic-based loading mechanism. 
Thermosensitivity of the NIPAM nanogels was believed to increase the drug loading capabilities of the nanogel by successive swelling and collapse cycles to maximize the penetration of R6G within the core $[42,52]$. However, for all three different incubation media and incubation temperatures, no significant difference on DL was found for each temperature cycle used as illustrated in figure S3. This result suggests that fast equilibration between inner or outer structure of the nanogels is reached independently of the loading protocol.

To confirm that the NIPAM nanogels act as affinity domains via electrostatic interactions from MAA component, the affinity was calculated as suggested by Grosberg et al. [53]. The affinity was derived from a Langmuir adsorption isotherm equation given below:

$D_{a d}=\frac{S \times K \times D_{s o l}}{K \times D_{s o l}+1}$

Where $D_{a d}$ is the concentration ( $\mathrm{mmol} / \mathrm{L}$ ) of R6G adsorbed on the nanogel, $D_{s o l}$ is the concentration (mmol/L) of free R6G remaining in solution, $S$ is the concentration (mmol/L) of maximum
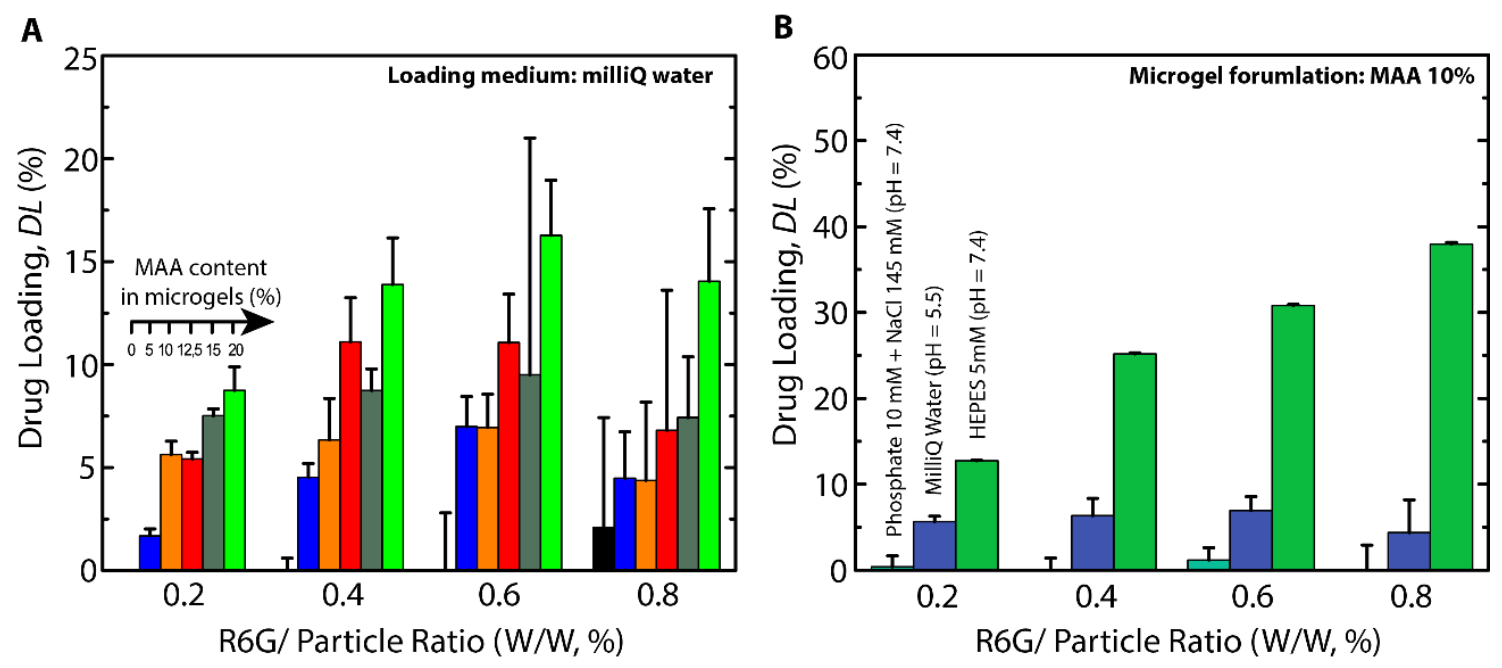

Figure 2. Characterization of R6G loading in NIPAM-co-MAA nanogels. Drug loadings from NIPAM nanogels containing 0 to $20 \%$ MAA loaded with R6G in pure water at different R6G / particle ratios (A). Drug loadings of R6G in MAA 10\% nanogels in different incubation media: PBS $10 \mathrm{mM}$ with $145 \mathrm{mM} \mathrm{NaCl}(\mathrm{pH}=7.4)$, pure water and HEPES $5 \mathrm{mM}$ without salt $(\mathrm{pH}=7.4)$ at different $\mathrm{R} 6 \mathrm{G} /$ particle ratios $(\mathrm{B})$. 
adsorption site and $K$ is the affinity constant of R6G to its adsorption site (L/mmol). We used Eq. 3 to extract the values of $S$ and $K$ (figure 3A) for each formulation, where $K$ was kept constant for MAA $>0 \%$.

The total affinity, $Q$, was defined as $Q=S \times K$. This value was plotted as a function of MAA content in the nanogel (figure 3B). The result shows a linear relationship, confirming the hypothesis that the affinity of R6G towards the NIPAM nanogels is only mediated by electrostatic interactions. Additionally, the calculated affinity $(Q)$ in HEPES was shown 6-fold $(6.4 \pm 0.5)$ higher than in pure water $(1.1 \pm 0.3)$, while in PBS $Q$ is not significant $(0.04 \pm 0.04)$ as illustrated in figure $3 \mathrm{C}$. These data clearly illustrate that the affinity of R6G was strongly modulated by the ionic strength of the medium and its $\mathrm{pH}$.

\subsection{In vitro release from hydrogels embedding liposomes}

The release behaviour of sulforhodamine B from liposomes embedded into hydrogels was investigated in HEPES buffer at pH 7.4 at $4{ }^{\circ} \mathrm{C}$ and $37{ }^{\circ} \mathrm{C}$ during $240 \mathrm{~h}$ (Figure 4).

The incorporation of liposomes into hydrogel produced a slower and controlled release of sulforhodamine B from all liposome formulations. In the case of the (50:50) formulation, an initial
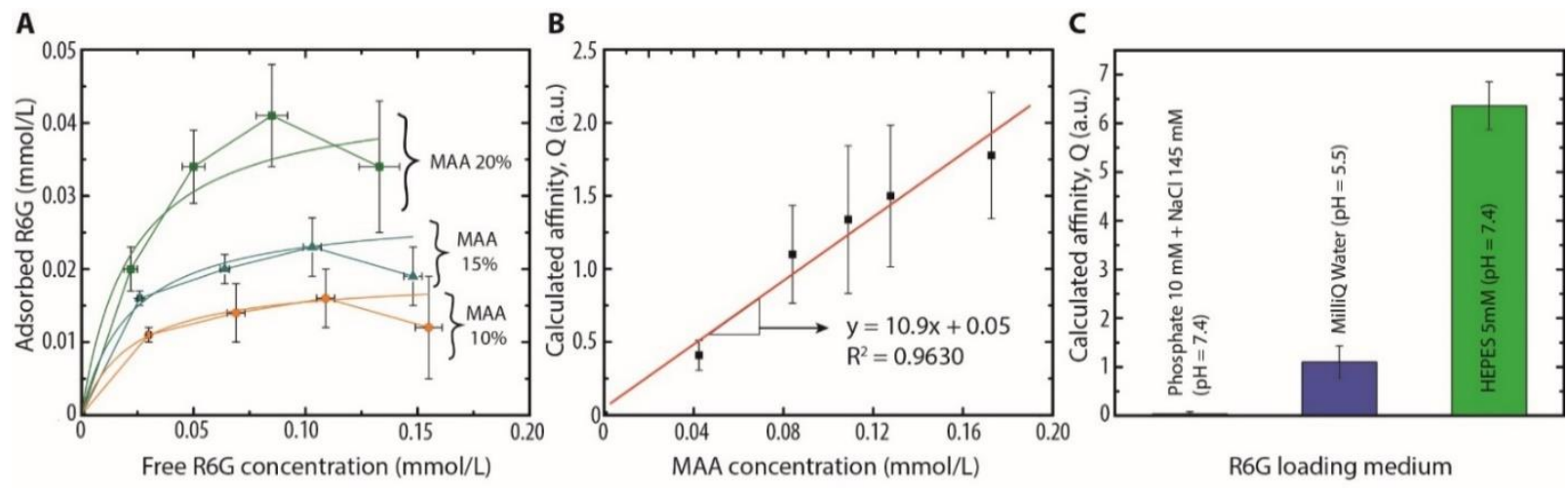

Figure 3. Langmuir isotherms (A) for 3 NIPAM nanogelss, where the affinity $Q$ was determined and plotted in function of MAA concentration (B). The affinity for R6G to NIPAM nanogels are also presented in three mediums and conditions (C). 
burst release was observed at both temperatures in contrast to the release from liposome suspensions (described in supplementary information). At $4{ }^{\circ} \mathrm{C}$, the cumulative release of sulforhodamine B started at $t=24 \mathrm{~h}$ with $30.4 \pm 2.8 \%$ of total sulforhodamine B and was very slow until $t=144 \mathrm{~h}$ when the release started to increase at cumulative release of $40.0 \pm 2.9 \%$. At $t=240 \mathrm{~h}$, only $52.4 \pm 2.9 \%$ of the total quantity of sulforhodamine B loaded within the hydrogel was released. Correspondingly, the (50:50) formulation showed great sensitivity towards the rise in temperature $\left(37^{\circ} \mathrm{C}\right)$ represented
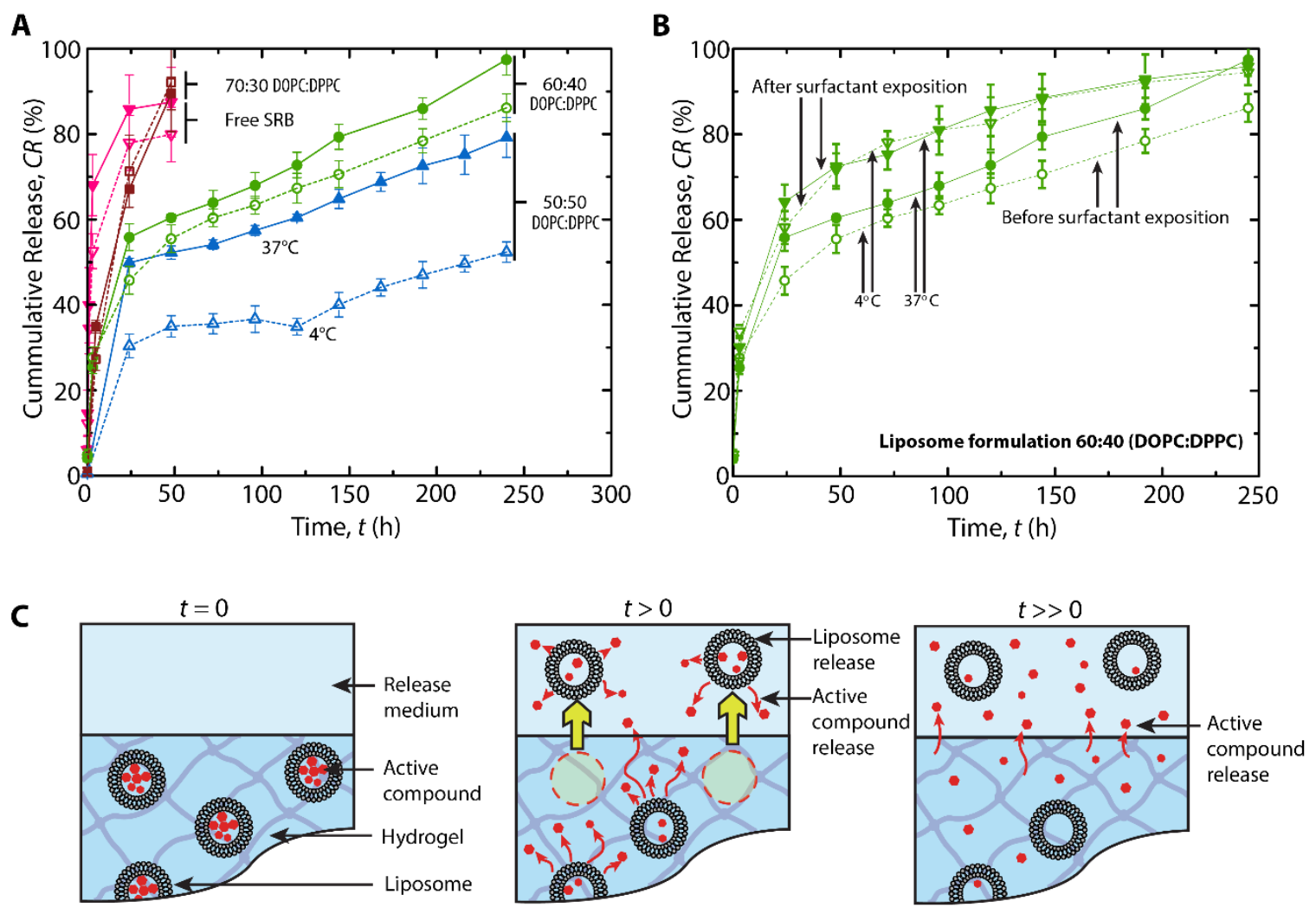

Figure 4. Release behavior of sulforhodamine B from hydrogels embedding liposomes. Sulforhodamine B release tested during 240 hours (A) from hydrogels embedding three different formulations (free SRB - reversed triangles, 70:30 DOPC:DPPC - squares, 60:40 DOPC:DPPC circles, 50:50 DOPC:DPPC - upright triangles) of liposomes at $4{ }^{\circ} \mathrm{C}$ (dashed lines) and $37^{\circ} \mathrm{C}$ (full lines). An increase in sulforhodamine B release rate from the (60:40) formulation following addition of $1 \% \mathrm{C} 12 \mathrm{E} 9$ surfactant, suggesting liposome release from the hydrogels at $4{ }^{\circ} \mathrm{C}$ and 37 ${ }^{\circ} \mathrm{C}$ for over 240 hours (B). Proposed release mechanism of liposomes and sulforhodamine B from hydrogels at different time intervals (C). 
by the change of the cumulative release profile compared to $4{ }^{\circ} \mathrm{C}$. After $24 \mathrm{~h}$ of incubation at $37^{\circ} \mathrm{C}$, $49.8 \pm 1.0 \%$ of sulforhodamine B were diffused from the hydrogel and continued to diffuse until $79.2 \pm 4.7 \%$ were released after $t=240 \mathrm{~h}$.

The release behaviour from hydrogels for the 60:40 formulation was also extended until $\mathrm{t}=240 \mathrm{~h}$ with superior control compared to liposomes alone. Similar to the 50:50 formulation, a significant burst release (30-50\%) was followed by a steady increased release rate every $24 \mathrm{~h}$ at both temperatures. However, with increased DOPC concentration in this formulation, the release was found less affected by temperature. The release at $37^{\circ} \mathrm{C}(97.5 \pm 3.6 \%$ after $t=240 \mathrm{~h})$ compared to the release at $4{ }^{\circ} \mathrm{C}(86.2 \pm 3.2 \%)$ after $t=240 \mathrm{~h}$ was only slightly faster for the former temperature.

Due to the same factors as in the (60:40) formulation, the release from hydrogels embedding the 70:30 formulation loaded with sulforhodamine B was very rapid but still slower than the release of free sulforhodamine B from the hydrogels. Those hydrogels released all their free sulforhodamine B content after $t=24 \mathrm{~h}$, while in case of the $70: 30$ formulation, the release was almost completed after $t=48 \mathrm{~h}$ at both $4{ }^{\circ} \mathrm{C}$ and $37^{\circ} \mathrm{C}$.

The burst release observed with each nanocomposite hydrogel formulations (figure 4A) was not found in liposome suspensions (figure S4). However, the UV light used to polymerize the hydrogels was tested on liposome suspensions and was found to cause leakage of sulforhodamine via oxidation or thermal heating of the lipids (supplementary information). Consequently, the burst observed can be attributed to the free sulforhodamine B in the hydrogel.

Finally, to verify whether intact liposomes have been released from the hydrogels, $10 \mu \mathrm{L}$ of $1 \%$ of nonaethylene glycol monododecyl ether (C12E9) detergent were added to the collected supernatants. Results showed an increase of the fluorescence intensity in each sample collected suggesting that the supernatants did not only contain free sulforhodamine B, but loaded liposomes as well. As seen in figure 4B, liposomes are already released from the hydrogel shortly after incubation started, which suggests that they were located close to hydrogel-medium interface as depicted in figure 4C. Diffusion of liposomes through the hydrogel network is facilitated by the particle and polymer network deformability. Indeed, 
liposome Young' modulus ( $3 \mathrm{kPa})$ confers high flexibility to the particle [54]. The hydrogels are expected to have a Young's modulus of a similar magnitude $(\sim 50 \mathrm{kPa})$, with a porosity of about $30-40 \mathrm{~nm}$ for this specific hydrogel formulation [55].

\subsection{In vitro release from hydrogel embedding NIPAM nanogels}

Release of R6G in a HEPES buffer (HEPES $5 \mathrm{mM}+\mathrm{NaCl} 145 \mathrm{mM}$ ) from NIPAM nanogels was studied over 72 hours at both $37^{\circ} \mathrm{C}$ and $4^{\circ} \mathrm{C}$ (figure 5). In each case, the release profiles presented two different processes. When the cumulative release was past a definite percentage (approximately $60-70 \%$ ), the release rate was drastically reduced (figure 5A). This "break point" was evaluated by linear extrapolation of both regimes (table S1).
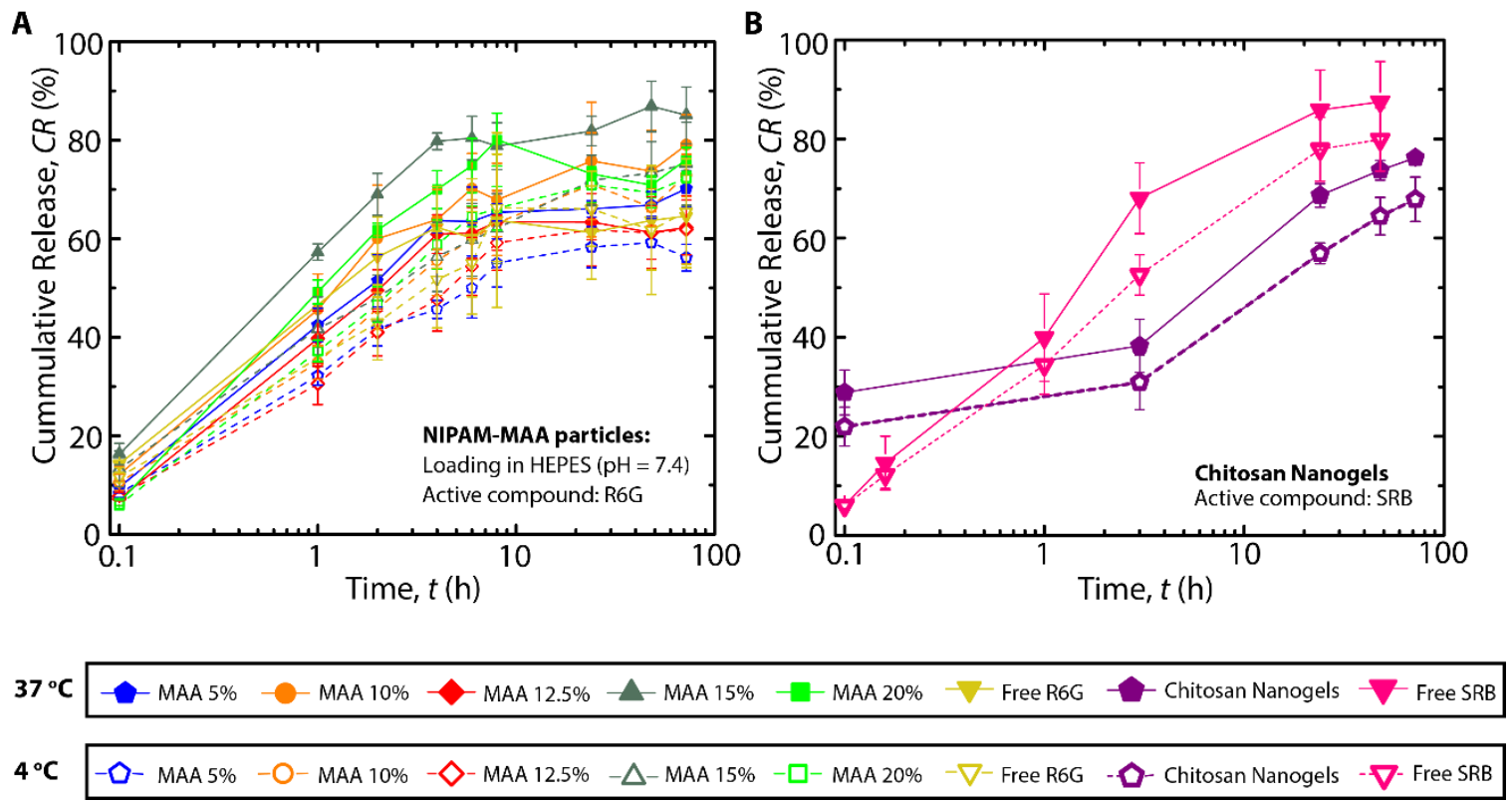

Figure 4. Release kinetics of nanogel formulations embedded in hydrogel structures at $4^{\circ} \mathrm{C}$ and $37^{\circ} \mathrm{C}$. Release kinetics of R6G were followed for 72 hours for each NIPAM nanogel formulation loaded with R6G in HEPES $5 \mathrm{mM}$ without salt (A) and release of sulforhodamine B from chitosan nanogels embedded in hydrogels (B). 
The loading medium of the NIPAM nanogels was first investigated, where loaded NIPAM nanogels in pure water were compared to loaded NIPAM nanogels in HEPES 5mM. With different drug loadings observed in the final hydrogels, it was expected that the final hydrogels would carry different quantities of affinity domains, resulting in a difference in release rates. Nanogels loaded in HEPES $5 \mathrm{mM}$ (figure 5A) initially released their content quickly, within 5 to 25 hours at $37^{\circ} \mathrm{C}$, releasing between 58-82\% R6G until reaching the "break-point". Similarly, nanogels loaded in pure water (figure S5) released $64-71 \%$ R6G in 4 to 8 hours. Finally, hydrogels containing free R6G initially released slightly faster in comparison to hydrogels embedding NIPAM nanogels at respective incubation temperatures, releasing their content in 4-8 hours (63-66\%). Despite a fast release at $37^{\circ} \mathrm{C}$, the hydrogels embedding NIPAM nanogels loaded in HEPES buffer demonstrated the ability to release their content over 10.7 hours, extending the release period compared to hydrogels containing free R6G, which released over $4.0 \mathrm{~h}$ until the "break point" was reached. Similarly, nanogels loaded in pure water also resulted in a "break point" at $8.3 \mathrm{~h}$. These results suggest that the R6G loading medium did not impact significantly on R6G release rate and mechanisms. Also, no clear correlation between the release rate and the MAA concentrations was found (figure 4A). However, the presence of the NIPAM nanogels suggests a slight reduction in the release rate. Those observations might be explained by the difference of affinity in the R6G loading medium (water / HEPES with no salt) in comparison with the release medium (buffered saline solution). The medium change might have drastically impacted the affinity of R6G to the nanogels, thus releasing without attractive force from the MAA groups, leaving no clear differences between formulations.

For the purpose of studying temperature effects and better understanding the release mechanisms, release profiles were also evaluated at $4^{\circ} \mathrm{C}$ for $72 \mathrm{~h}$ (figures $4 \mathrm{~A}$ dotted lines and S5). Under those conditions, R6G was initially released up to $58-72 \%$ for each formulation, typically releasing within 11 - $25 \mathrm{~h}$ (table 2). Comparatively, the nanogels loaded in pure water released 64-69\% of their total content in 7-9 hours and free R6G released $65.8 \%$ within $7.6 \mathrm{~h}$ until reaching the "break point". 
Release at $4^{\circ} \mathrm{C}$ additionally supports the hypothesis of increased sustained release using NIPAM nanogels which exhibited an additional 3-17 extra hours of release (nanogels loaded in HEPES for instance) comparatively to free R6G. This can be explained by the viscosity of the buffered saline at $4^{\circ} \mathrm{C}$, decreasing the $\mathrm{R} 6 \mathrm{G}$ diffusion through the hydrogel network. Additionally, it is possible that the NIPAM nanogel thermosensitivity has influenced the R6G release rates. Since the embedding hydrogel is polymerized at room temperature, the NIPAM nanogels are trapped in their swollen state, but at temperature of $37^{\circ} \mathrm{C}$ (release temperature), the NIPAM nanogels collapse, expelling R6G and leaving possible micropores within the hydrogel, thus facilitating further R6G release.

\subsection{Release from hydrogel embedding chitosan nanogels}

Release from nanogels of chitosan and HA was performed by the same method. Release of sulforhodamine was evaluated at both $4^{\circ} \mathrm{C}$ and $37^{\circ} \mathrm{C}$. The resulting release kinetics represented in figure 4B was compared to hydrogel containing free sulforhodamine B). Chitosan nanogels released sulforhodamine B in a more controlled fashion than free sulforhodamine B. For instance, nanogels released $73.7 \pm 2.0 \%$ over a period of $48 \mathrm{~h}$ at $37^{\circ} \mathrm{C}$ and $64.4 \pm 3.8 \%$ in the same timeframe at $4{ }^{\circ} \mathrm{C}$, whereas the same amount of free sulforhodamine B was released twice as fast (table S1). Although, a high burst effect (21-29\%) was observed initially at the first time-point, suggesting an immediate release of sulforhodamine B upon swelling in the incubation medium. Once more, exposure to low temperature decreased the release rate by reducing to $9 \%$ the cumulative sulforhodamine B release at $4^{\circ} \mathrm{C}$. Such results suggest that the nanogel inclusions significantly impacted on the release profiles of the hydrogel by altering its release pattern in a controlled fashion.

\subsection{Comparison of nanogel-based and liposome-based systems}

The choice of two different drug models was motivated by the encapsulation mechanism. Indeed, both model drugs are encapsulated via electrostatic forces. Sulforhodamine B is negatively charged 
at $\mathrm{pH}=7.4(\mathrm{pka}=4.2)$ which favors its encapsulation in chitosan-based nanogels. On the other hand, rhodamine $6 \mathrm{G}$ is positively charged at physiological $\mathrm{pH}(\mathrm{pKa}=10)$ which facilitates its encapsulation in negatively charged microgels. Due to the higher solubility of sulforhodamine B compared to rhodamine $6 \mathrm{G}$, the former was chosen as a model drug for the liposome-based formulations.

In order to identify key differences in the release mechanisms between the different systems tested in this study, we renormalized the release profiles using the non-dimensional time $t^{*}$ defined as:[35] $t^{*}=\frac{t}{\frac{L^{2}}{D} \times\left(1+K \times C_{N P}\right)}$

Where $t$ is the release time (in hours), $L$ the thickness of the hydrogel (in $\mathrm{cm}$ ), $D$ the effective diffusion coefficient $\left(\mathrm{cm}^{2} / \mathrm{s}\right), K$ the affinity constant $\left(\mathrm{mM}^{-1}\right)$ of the drug to the particle and $\mathrm{C}_{\mathrm{NP}}$ the concentration of particles / binding sites $(\mathrm{mM})$.

For the liposome-based systems, we considered that $\mathrm{K}<<1$ which simplified the expression of $t^{*}$. The effective diffusion coefficient $D_{\text {eff }}$ of drug is determined using Fick's second law [54]:
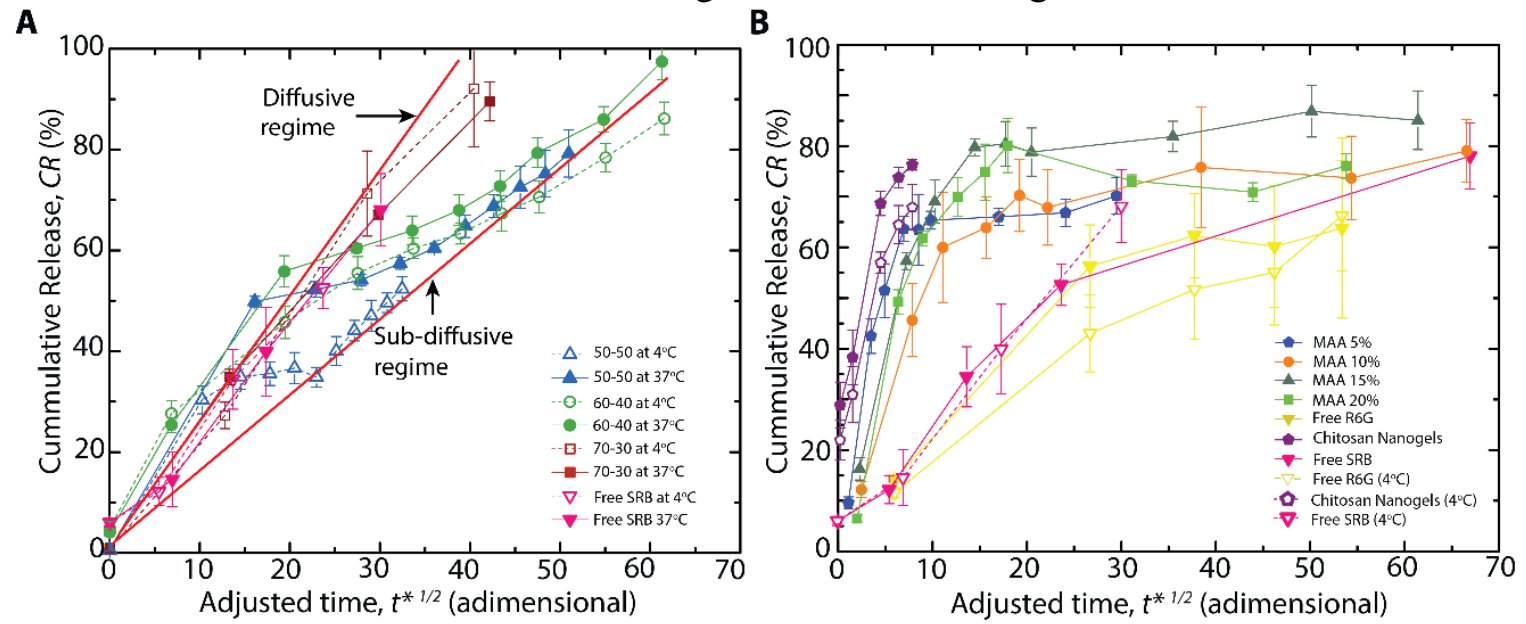

Figure 6. Release profiles with time mathematically adjusted with (Eq.4) to compare both nano-inclusions of liposomes (A) and affinity-based hydrogels containing nanogels (B). In comparison with their respective unadjusted release curves, an effect of superposition should be created with both adjustments. For clarity purposes, only representative NIPAM nanogels are shown for a better trend visualization. 
$C R \%=2 \times\left(\frac{D_{e f f} \times t}{\pi \times L^{2}}\right)^{1 / 2}$

Where $C R \%$ corresponds to the cumulative release of the drug. For the liposome-based systems (Figure 6A), the release profile follows two linear (therefore purely diffusive) regimens. The first one, which is superimposed to the release of the free drug, corresponds to the release of mobile liposomes and free drug in the proximal region of the interface. The second regimen (called subdiffusive regimen), which corresponds to the diffusion of the drug through the liposome membrane and the hydrogel network, is quickly reached for all the tested formulations. These findings also support the proposed release mechanisms with nanocomposite hydrogels made from liposomes, depicted in figure $4 \mathrm{C}$, where the release is performed in two distinct steps.

Using the same renormalization of the data, NIPAM nanogels exhibited a strikingly different behavior (Figure 6B). These affinity-based systems also present an initial burst release mostly dominated by diffusion which stops abruptly after reaching approximately $65 \%$ of release. After this point, all the NIPAM nanogels-embedded hydrogels seem to slowly evolve towards a subdiffusive regimen, not reached in our experiments.

Finally, normalisation of chitosan nanogels proved to be a relatively similar system to the NIPAM nanogel system (Figure 6B). In a similar fashion, nanogels despite containing important quantity of polyelectrolytes also proved to be massively affected by salts within the release medium. Although, one difference is observable with the drug used. While SRB had a very similar diffusion rate as R6G (widely known in water), the latter seemed to adsorb slightly more importantly to the hydrogel matrix than SRB, which can be seen at approximately $65 \%$ of cumulative release. This latter point also suggests the presence of the second sub-diffusive release step whilst using NIPAM nanogels and its absence in nanogels.

Liposome nano-inclusions proved to be very efficient to prolong the release period as demonstrated in figure 4. However, the use of NIPAM or chitosan nanogels nano-inclusion as an affinity-based release systems seemed to be limited by the presence of salt ions. A possible way to counter balance 
this effect could be by increasing the quantity of particles $[15,55]$, much higher than what we used in this study.

\section{Conclusion}

In this study, we were able to observe and clearly identify different release mechanisms for each nanoparticle formulation embedded in hydrogels. The combination of liposomes and hydrogels provided the best controlled release of the active, due to its two diffusive regimens: the first diffusive regimen was characterized by free sulforhodamine B dispersed within the hydrogel matrix and the second "sub-diffusive" regimen characterized by sulforhodamine B entrapped within liposomes. Nanogels made of NIPAM, however, did not impact significantly the release of R6G due to salt interactions from the release medium with the affinity domains. Two release mechanisms were also identified: first, the release of free R6G of the hydrogel matrix (burst) and then a subdiffusive regimen where R6G bound to the hydrogel matrix slowly diffuses into the external medium. As for chitosan nanogels, they exhibited a similar release profile as to NIPAM nanogels minus the second sub-diffusive regimen due to the choice of the released molecule. Overall, we have precisely described the release mechanisms of each nanocomposite hydrogel formulation we developed. In the light of these results, it is possible to assert that, to achieve controlled release, the diffusion-based mechanism is by far superior, while the affinity-based one, such as electrostatic interactions, could still need further improvement as a drug delivery system within a biological system. We still believe that the affinity-based ones hold significant potential in pharmaceutical applications and in nanomedicine due to their ability to tune finely the affinity domains.

\section{Acknowledgements}

Xavier Banquy acknowledges the financial support of NSERC and CIHR through the Canadian Research Chair Program. Pierre-Luc Latreille is grateful for financial support of GRUM, Faculty of Pharmacy, FRQ-NT and NSERC. 


\section{References}

[1] A.Z. Wang, F. Gu, L. Zhang, J.M. Chan, A. Radovic-Moreno, M.R. Shaikh, R.S. Langer, O.C. Farokhzad, Biofunctionalized Targeted Nanoparticles for Therapeutic Applications, Expert Opin. Biol. Ther., 8 (2008) 1063-1070.

[2] R.M. Gant, A.A. Abraham, Y. Hou, B.M. Cummins, M.A. Grunlan, G.L. Coté, Design of a self-cleaning thermoresponsive nanocomposite hydrogel membrane for implantable biosensors, Acta Biomater., 6 (2010) 2903-2910.

[3] S. Mourtas, S. Fotopoulou, S. Duraj, V. Sfika, C. Tsakiroglou, S.G. Antimisiaris, Liposomal drugs dispersed in hydrogels: Effect of liposome, drug and gel properties on drug release kinetics, Colloids Surf. B. Biointerfaces, 55 (2007) 212-221.

[4] Y. Liang, K.L. Kiick, Liposome-Cross-Linked Hybrid Hydrogels for Glutathione-Triggered Delivery of Multiple Cargo Molecules, Biomacromolecules, 17 (2016) 601-614.

[5] K.E. Schlichting, T.M. Copeland-Johnson, M. Goodman, R.J. Lipert, T. Prozorov, X. Liu, T.O. McKinley, Z. Lin, J.A. Martin, S.K. Mallapragada, Synthesis of a novel photopolymerized nanocomposite hydrogel for treatment of acute mechanical damage to cartilage, Acta Biomater., 7 (2011) 3094-3100.

[6] N. Sahiner, M. Singh, In situ micro/nano-hydrogel synthesis from acrylamide derivates with lecithin organogel system, Polymer, 48 (2007) 2827-2834.

[7] N. Sahiner, W.T. Godbey, G.L. McPherson, V.T. John, Microgel, nanogel and hydrogel-hydrogel semi-IPN composites for biomedical applications: synthesis and characterization, Colloid. Polym. Sci., 284 (2006) 1121-1129.

[8] L.-W. Xia, R. Xie, X.-J. Ju, W. Wang, Q. Chen, L.-Y. Chu, Nano-structured smart hydrogels with rapid response and high elasticity, Nat. Commun., 4 (2013) 2226.

[9] S. Grijalvo, J. Mayr, R. Eritja, D.D. Diaz, Biodegradable liposome-encapsulated hydrogels for biomedical applications: a marriage of convenience, Biomaterials Science, 4 (2016) 555-574.

[10] C. Silan, A. Akcali, M.T. Otkun, N. Ozbey, S. Butun, O. Ozay, N. Sahiner, Novel hydrogel particles and their IPN films as drug delivery systems with antibacterial properties, Colloids Surf. B. Biointerfaces, 89 (2012) 248-253.

[11] N. Sahiner, Hydrogels of Versatile Size and Architecture for Effective Environmental Applications Turk. J. Chem., 32 (2008) 113-123.

[12] P. Thoniyot, M.J. Tan, A.A. Karim, D.J. Young, X.J. Loh, Nanoparticle-Hydrogel Composites: Concept, Design, and Applications of These Promising, Multi-Functional Materials, Advanced Science, 2 (2015) 1400010-n/a.

[13] C.A. Carmona-Moran, O. Zavgorodnya, A.D. Penman, E. Kharlampieva, S.L. Bridges Jr, R.W. Hergenrother, J.A. Singh, T.M. Wick, Development of gellan gum containing formulations for transdermal drug delivery: Component evaluation and controlled drug release using temperature responsive nanogels, Int. J. Pharm., 509 (2016) 465-476.

[14] Y. Tang, Y. Zhao, Y. Li, Y. Du, A thermosensitive chitosan/poly(vinyl alcohol) hydrogel containing nanoparticles for drug delivery, Polym. Bull., 64 (2010) 791-804.

[15] D. Sivakumaran, D. Maitland, T. Hoare, Injectable Microgel-Hydrogel Composites for Prolonged Small-Molecule Drug Delivery, Biomacromolecules, 12 (2011) 4112-4120.

[16] G.E. Cinay, P. Erkoc, M. Alipour, Y. Hashimoto, Y. Sasaki, K. Akiyoshi, S. Kizilel, Nanogel-integrated pH Responsive Composite Hydrogels for Controlled Drug Delivery, ACS Biomaterials Science \& Engineering, (2017).

[17] A. Laouini, C. Jaafar-Maalej, I. Limayem-Blouza, S. Sfar, C. Charcosset, H. Fessi, Preparation, Characterization and Applications of Liposomes: State of the Art, J. Colloid Sci. Biotechnol., 1 (2012) 147-168.

[18] H. Sadozai, D. Saeidi, Recent Developments in Liposome-Based Veterinary Therapeutics, ISRN Vet. Sci., 2013 (2013) 8.

[19] A.D. Bangham, J. De Gier, G.D. Greville, Osmotic properties and water permeability of phospholipid liquid crystals, Chem. Phys. Lipids, 1 (1967) 225-246.

[20] Y. Guan, Y. Zhang, PNIPAM microgels for biomedical applications: from dispersed particles to 3D assemblies, Soft Matter, 7 (2011) 6375-6384.

[21] R.H. Pelton, P. Chibante, Preparation of aqueous latices with N-isopropylacrylamide, Colloids Surf., 20 (1986) $247-256$.

[22] A.K. Saikia, S. Aggarwal, U.K. Mandal, Preparation and Controlled Drug Release Characteristics of Thermoresponsive PEG/Poly (NIPAM-co-AMPS) Hydrogels, International Journal of Polymeric Materials and Polymeric Biomaterials, 62 (2012) 39-44.

[23] T. Hoare, R. Pelton, Impact of Microgel Morphology on Functionalized Microgel-Drug Interactions, Langmuir, 24 (2008) 10051012.

[24] X. Hu, Z. Tong, L.A. Lyon, Control of poly(N-isopropylacrylamide) microgel network structure by precipitation polymerization near the lower critical solution temperature, Langmuir, 27 (2011) 4142-4148.

[25] T.A. Sonia, C. Sharma, Chitosan and Its Derivatives for Drug Delivery Perspective, in: R. Jayakumar, M. Prabaharan, R.A.A. Muzzarelli (Eds.) Chitosan for Biomaterials I, Springer Berlin Heidelberg2011, pp. 23-53.

[26] G. Berth, H. Dautzenberg, The degree of acetylation of chitosans and its effect on the chain conformation in aqueous solution, Carbohydr. Polym., 47 (2002) 39-51.

[27] M.W. Anthonsen, K.M. Varum, O. Smidsrod, Solution Properties of Chitosans - Conformation and Chain Stiffness of Chitosans with Different Degrees of N-Acetylation, Carbohydr. Polym., 22 (1993) 193-201.

[28] C. Schatz, C. Viton, T. Delair, C. Pichot, A. Domard, Typical Physicochemical Behaviors of Chitosan in Aqueous Solution, Biomacromolecules, 4 (2003) 641-648. 
[29] P. Sorlier, A. Denuzière, C. Viton, A. Domard, Relation between the Degree of Acetylation and the Electrostatic Properties of Chitin and Chitosan, Biomacromolecules, 2 (2001) 765-772.

[30] P. Sorlier, C. Rochas, I. Morfin, C. Viton, A. Domard, Light Scattering Studies of the Solution Properties of Chitosans of Varying Degrees of Acetylation, Biomacromolecules, 4 (2003) 1034-1040.

[31] S.A. Agnihotri, N.N. Mallikarjuna, T.M. Aminabhavi, Recent advances on chitosan-based micro- and nanoparticles in drug delivery, J Control Release, 100 (2004) 5-28.

[32] J.K. Francis Suh, H.W.T. Matthew, Application of chitosan-based polysaccharide biomaterials in cartilage tissue engineering: a review, Biomaterials, 21 (2000) 2589-2598.

[33] N. Hanauer, P.L. Latreille, S. Alsharif, X. Banquy, 2D, 3D and 4D active compound delivery in tissue engineering and regenerative medicine, Curr. Pharm. Des., 21 (2015) 1506-1516.

[34] M. Goldberg, R. Langer, X. Jia, Nanostructured materials for applications in drug delivery and tissue engineering, J. Biomater. Sci. Polym. Ed., 18 (2007) 241-268.

[35] K. Vulic, M.M. Pakulska, R. Sonthalia, A. Ramachandran, M.S. Shoichet, Mathematical model accurately predicts protein release from an affinity-based delivery system, J. Control. Release, 197 (2015) 69-77.

[36] K. Vulic, M.S. Shoichet, Affinity-Based Drug Delivery Systems for Tissue Repair and Regeneration, Biomacromolecules, 15 (2014) 3867-3880.

[37] P.-A. Monnard, T. Oberholzer, P. Luisi, Entrapment of nucleic acids in liposomes, Biochim. Biophys. Acta, 1329 (1997) $39-50$.

[38] T. Courant, V.G. Roullin, C. Cadiou, M. Callewaert, M.C. Andry, C. Portefaix, C. Hoeffel, M.C. de Goltstein, M. Port, S. Laurent, L.V. Elst, R. Muller, M. Molinari, F. Chuburu, Hydrogels Incorporating GdDOTA: Towards Highly Efficient Dual T1/T2 MRI Contrast Agents, Angew. Chem. Int. Ed., 51 (2012) 9119-9122.

[39] Q. Gan, T. Wang, C. Cochrane, P. McCarron, Modulation of surface charge, particle size and morphological properties of chitosan-TPP nanoparticles intended for gene delivery, Colloids Surf. B. Biointerfaces, 44 (2005) 65-73.

[40] T. López-León, J.L. Ortega-Vinuesa, D. Bastos-González, A. Elaïssari, Cationic and Anionic Poly(N-isopropylacrylamide) Based Submicron Gel Particles: Electrokinetic Properties and Colloidal Stability, J. Phys. Chem. B, 110 (2006) 4629-4636.

[41] B. Ku, H. Seo, B. Chung, Synthesis and characterization of thermoresponsive polymeric nanoparticles, BioChip J, 8 (2014) 814.

[42] J. Kleinen, A. Klee, W. Richtering, Influence of architecture on the interaction of negatively charged multisensitive poly(Nisopropylacrylamide)-co-methacrylic acid microgels with oppositely charged polyelectrolyte: absorption vs adsorption, Langmuir, 26 (2010) 11258-11265.

[43] J. Baier Leach, K.A. Bivens, C.W. Patrick Jr, C.E. Schmidt, Photocrosslinked hyaluronic acid hydrogels: Natural, biodegradable tissue engineering scaffolds, Biotechnol. Bioeng., 82 (2003) 578-589.

[44] F.A. Oyarzun-Ampuero, J. Brea, M.I. Loza, D. Torres, M.J. Alonso, Chitosan-hyaluronic acid nanoparticles loaded with heparin for the treatment of asthma, Int. J. Pharm., 381 (2009) 122-129.

[45] H. Katas, C. Mui Wen, Preparation and Characterisation of Highly Loaded Fluorescent Chitosan Nanoparticles, ISRN Pharmaceutics, 2011 (2011) 5.

[46] M. de la Fuente, B. Seijo, M.J. Alonso, Novel Hyaluronan-Based Nanocarriers for Transmucosal Delivery of Macromolecules, Macromol. Biosci., 8 (2008) 441-450.

[47] A. Almalik, R. Donno, C.J. Cadman, F. Cellesi, P.J. Day, N. Tirelli, Hyaluronic acid-coated chitosan nanoparticles: Molecular weight-dependent effects on morphology and hyaluronic acid presentation, J. Control. Release, 172 (2013) 1142-1150.

[48] T. Hoare, R. Pelton, Functionalized Microgel Swelling: Comparing Theory and Experiment, J. Phys. Chem. B, 111 (2007) 1189511906.

[49] H. Dong, H. Du, X. Qian, Theoretical Prediction of pKa Values for Methacrylic Acid Oligomers Using Combined Quantum Mechanical and Continuum Solvation Methods, J. Phys. Chem. A, 112 (2008) 12687-12694.

[50] J.M. Giussi, M.I. Velasco, G.S. Longo, R.H. Acosta, O. Azzaroni, Unusual temperature-induced swelling of ionizable poly(Nisopropylacrylamide)-based microgels: experimental and theoretical insights into its molecular origin, Soft Matter, 11 (2015) 8879-8886.

[51] J. Zhou, J. Wei, T. Ngai, L. Wang, D. Zhu, J. Shen, Correlation between Dielectric/Electric Properties and Cross-Linking/Charge Density Distributions of Thermally Sensitive Spherical PNIPAM Microgels, Macromolecules, 45 (2012) 6158-6167.

[52] A.V. Kabanov, S.V. Vinogradov, Nanogels as Pharmaceutical Carriers: Finite Networks of Infinite Capabilities, Angew. Chem., 48 (2009) 5418-5429.

[53] K. Ito, J. Chuang, C. Alvarez-Lorenzo, T. Watanabe, N. Ando, A.Y. Grosberg, Multiple point adsorption in a heteropolymer gel and the Tanaka approach to imprinting: experiment and theory, Prog. Polym. Sci., 28 (2003) 1489-1515.

[54] R. Pjanović, N. Bošković-Vragolović, J. Veljković-Giga, R. Garić-Grulović, S. Pejanović, B. Bugarski, Diffusion of drugs from hydrogels and liposomes as drug carriers, J. Chem. Technol. Biotechnol., 85 (2010) 693-698.

[55] D. Sivakumaran, D. Maitland, T. Oszustowicz, T. Hoare, Tuning drug release from smart microgel-hydrogel composites via cross-linking, J. Colloid Interface Sci., 392 (2013) 422-430. 